
\title{
25 Research Soure \\ Lactobacillus Plantarum LRCC5314 Includes a Gene for a Substance that Stimulates the Secretion of Serotonin
}

Jiseon Jeong

Chung-Ang University College of Medicine

Seokmin Yoon

Lotte R\&D Center

Jong-Hwa Kim

Chung-Ang University College of Medicine

Wonyong Kim ( $\nabla$ kimwy@cau.ac.kr)

Chung-Ang University College of Medicine

\section{Research Article}

Keywords: Lactic acid bacteria, Lactobacillus plantarum LRCC5314, Probiotics, Whole-genome analysis, Tryptophan, Serotonin

Posted Date: June 21st, 2021

DOI: https://doi.org/10.21203/rs.3.rs-622010/v1

License: (c) (i) This work is licensed under a Creative Commons Attribution 4.0 International License. Read Full License 


\section{Abstract}

Background: As the functions of probiotics within the same species may not be shared, it is important to analyze the genetic characteristics of strains to determine their safety and usefulness before industrial applications.

Results: The complete genome of Lactobacillus plantarum LRCC5314 isolated from kimchi was acquired through PacBio sequencing to confirm probiotic functions and genetic characteristics. Phylogenetic and comparative genomic analyses were performed between $L$. plantarum LRCC5314 and L. plantarum ATCC $14917^{\top}$, and the results showed that two strains were highly similar. However, there were some genetic differences. The characteristics of carbon source metabolism were also determined based on the KEGG database and physiological properties. L. plantarum LRCC5314 could metabolize hexoses through homofermentation, which produces only lactic acid. According to gene annotation, L. plantarum LRCC5314 could encode almost complete biosynthetic pathway to produce tryptophan, which can be used as a precursor of serotonin. The serotonin ELISA test using Caco-2 and HT-22 cells treated with a supernatant of the bacterial culture showed that more serotonin was produced by treated cells than by untreated cells.

Conclusion: L. plantarum LRCC5314 could provide a source for serotonin production, and L. plantarum LRCC5314 could be used as a functional probiotic for stress regulation.

\section{Background}

The genus Lactobacillus is a type of lactic acid bacteria (LAB) with diverse species. These rod-shaped Gram-positive bacteria are found in various environments, such as silage, fermented foods, fecal samples [1-3], and the human body, including the gastrointestinal tract and oral cavity [4,5]. Species of the genus Lactobacillus are widely used to manufacture dairy products, such as cheese, yogurt, and kefir starter [68]. In addition, some strains of lactobacilli, including L. rhamnosus, L. plantarum, L. casei, and $L$. raffinolactis, not only have fermentation functions but also have beneficial effects on human health as consumable probiotics.

According to the Food and Agriculture Organization/World Health Organization (FAO/WHO), probiotics are defined as "live microorganisms that, when administered in adequate amounts, confer a health benefit on the host" [9]. Probiotics modulate the gut environment by balancing the microflora. Several LAB, such as lactobacilli or lactococci, are recognized as probiotics, as they have different functions, such as lowering cholesterol or controlling immune reactions $[10,11]$. Over recent decades, L. plantarum and $L$. rhamnosus have been investigated for their beneficial effects on diabetes, obesity, liver disorder, and stress $[12,13]$. These days, along with medical supplies, probiotics are used to improve and prevent health problems.

However, comparative studies among probiotic strains have shown that the features of probiotics and their corresponding health benefits are strain-specific. These benefits may not be generalizable and may 
not be shared among strains, even among those of the same species [14]. For this reason, the genomic characterization of probiotics is important for the study of beneficial functions or mechanisms. Moreover, genome-based analysis is required to investigate the stability and safety of probiotics for industrial applications. Probiotics also exert negative effects through undisclosed genes. Virulence or antibiotic resistance genes can be transferred to other bacteria from probiotics via horizontal gene transfer [15]. Whole-genome analysis of probiotic strains can prevent potential risks by predicting them and thus increasing utilization efficiency.

L. plantarum LRCC5314 was isolated from kimchi as a probiotic candidate. In this study, we investigated the whole genome of L. plantarum LRCC5314 to characterize the strain via comparative, genome content, and metabolic pathway analyses. In addition, we identified the functional factors of $L$. plantarum LRCC5314 through gene annotation and provided the molecular biological evidence for its practical use as a probiotic strain.

\section{Materials And Methods}

\section{Phylogenetic and physiological characteristics of L. plantarum LRCC5314}

The genomic DNA of L. plantarum LRCC5314 was extracted using the genomic G-spin DNA extraction kit for bacteria (Intron, Seongnam, Korea). The sequence of $16 \mathrm{~s}$ rRNA was amplified by polymerase chain reaction (PCR) with the universal primers $8 \mathrm{~F}$ and 1525R [33] and purified using the Accuprep PCR purification kit (Bioneer, Seoul, Korea). The purified gene was sequenced directly, and the sequence similarity levels among closely related strains were calculated with EzBioCloud (https://www.ezbiocloud.net/identify) [34]. The phylogenetic distance between L. plantarum LRCC5314 and related strains of the genus Lactobacillus was calculated using CLUSTAL-X 2.1 software [35]. The phylogenetic trees were constructed using the neighbor-joining, maximum-parsimony, and maxi-mumlikelihood methods with MEGA 7 software [35-38]. To determine the physiological characteristics of $L$. plantarum LRCC5314, API 50CH, API 20E, and API ZYM (Biomerieux, Marcy-l'Étoile, France) tests were performed according to the manufacturer's instructions.

\section{Whole-genome sequencing and annotation}

The whole genome of L. plantarum LRCC5314 was sequenced using the PacBio RS $\otimes$ platform with the $20 \mathrm{~kb}$ SMRTbell TPK library kit. The sequences were assembled de novo with RS HGAP Assembly (version 3.0) in PacBio SMRT Analysis software (version 2.3). Genomic annotation was performed using the RAST server (version 2.0) [39], and the National Center for Biotechnology Information (NCBI)'s Prokaryotic Genomes Annotation Pipeline 4.1 [40] was used to combine the results. WebMGA on-line tools (version 2.2.15; http://weizhong-lab.ucsd.edu/webMGA/) [41] were used to group protein functions with the COG database. Antimicrobial resistance genes were identified using the program ResFinder (version 4.1; https://cge.cbs.dtu.dk/services/ResFinder/) [42]. The carbon source metabolic pathway was constructed based on the Kyoto Encyclopedia of Genes and Genomes (KEGG) database [43]. The sequences 
generated in this study were deposited in the DDBJ/EMBL/GenBank International Nucleotide Sequence Database under the BioProject ID PRJNA716786.

\section{Metabolic pathway analysis of carbon sources}

Based on KEGG pathway and protein BLAST [44] analyses, the carbon metabolic pathways of $L$. plantarum LRCC5314 were constructed. The pyruvate, pentose phosphate, sucrose, lactose, and galactose metabolism pathways of the strain were mapped according to the KEGG pathway. To construct a pathway, only mapped genes were used. Protein BLAST was used to reconfirm the functions of the characteristic genes.

\section{Comparative genomic analysis of $L$. plantarum LRCC5314}

The whole-genome sequence of $L$. plantarum ATCC $14917^{\top}$ (GCF_000143745) was obtained from NCBI GenBank for comparative genomic analysis with L. plantarum LRCC5314. The digital DNA-DNA hybridization (dDDH) value with L. plantarum ATCC 14917T was calculated using the Genome-toGenome Distance Calculator (GGDC; version 2.1; http://ggdc.dsmz.de/ggdc.php) [45]. OrthoANI values between L. plantarum LRCC5314 and related strains in the genus Lactobacillus were calculated using Orthologous Average Nucleotide Identity Tool (OAT) software [46]. A circular map to compare the genome sequence between L. plantarum LRCC5314 and L. plantarum ATCC $14917^{\top}$ was created using Blast Ring Image Generator (BRIG) software [47]. CRISPRFinder [48] was used to analyze clustered regularly interspaced palindromic repeats (CRISPRs).

\section{Production of bacterial supernatant}

L. plantarum LRCC5314 was cultured at $30^{\circ} \mathrm{C}$ under aerobic conditions in De Man, Rogosa and Sharpe (MRS) broth (BD Difco, Franklin Lakes, NJ, USA) overnight. After reaching an OD of 1.0 at $600 \mathrm{~nm}$, the cultured broth was centrifuged for $10 \mathrm{~min}$ at $8,000 \times \mathrm{g}$. The supernatant was filtered with a $0.22 \mu \mathrm{m}$ filter after harvesting and kept at $-20^{\circ} \mathrm{C}$ until use.

\section{Cell culture}

The cell lines Caco-2 and HT-22 were purchased from the American Type Culture Collection (ATCC; Manassas, VA, USA) and Merck (Darmstadt, Germany), respectively. Caco-2 cells were cultured in Minimum Essential Medium (MEM; Gibco, Waltham, MA, USA) supplemented with $10 \%$ fetal bovine serum and 1\% penicillin. HT-22 cells were cultured in Dulbecco's Modified Eagle Medium (DMEM; Lonza, Basel, Switzerland) supplemented with $10 \%$ fetal bovine serum and $1 \%$ penicillin. The cell lines were incubated at $37^{\circ} \mathrm{C}$ with $5 \% \mathrm{CO}_{2}$.

\section{Measurement of serotonin release}

The cultured Caco-2 and HT-22 cells were seeded in 24 -well plates $(1 \times 105$ cells/well $)$ and incubated until sub-confluent. Then, the cells were treated with the bacterial super-natant and incubated for $1 \mathrm{~h}$ at $37^{\circ} \mathrm{C}$. 
The supernatants of the treated wells were collected after incubation and centrifuged at $1,000 \times \mathrm{g}$ for 3 min. The serotonin content of the cell supernatants was quantified with the Serotonin High Sensitive ELISA kit (IBL, Hamburg, Germany) according to the manufacturer's instructions.

\section{Statistical analysis}

Multiple comparisons were performed by t-test and GraphPad Prism (v.6.0.1) (Graph Pad Software Inc., La Jolla, CA, USA). All data are presented as the mean \pm standard error of the mean (SEM), and $p$ values < 0.05 were considered significant.

\section{Results}

\section{Phylogenetic and phenotypic features of the isolated LAB strain}

The 16S rRNA gene of L. plantarum LRCC5314 was obtained and compared with available reference sequences from the GenBank database and EzBioCloud (http://www.ezbiocloud.net/eztaxon).

Phylogenetic analysis based on the 16S rRNA gene showed that $L$. plantarum LRCC5314 was clustered with the L. plantarum species (Fig. 1). L. plantarum LRCC5314 was the most closely related to $L$. plantarum ATCC $14917^{\top}$ with a similarity of $100 \%$, followed by L. pentosus $(99.9 \%)$, L. paraplantarum (99.7\%), and L. daowaiensis (99.1\%). The results showed that the isolated LRCC5314 strain was a strain of L. plantarum.

According to the API test, L. plantarum LRCC5314 could utilize glycerol, L-arabinose, D-ribose, Dgalactose, D-glucose, D-fructose, D-mannose, D-mannitol, D-sorbitol, $\mathrm{N}$-acetylglucosamine, amygdalin, arbutin, esculin, salicin, D-cellobiose, D-maltose, D-melibiose, D-saccharose, D-trehalose, D-melezitose, gentiobiose, D-furanose, potassium gluconate, and potassium 5-ketogluconate. L. plantarum LRCC5314 could produce acetoin from sodium pyruvate and was negative for $\mathrm{NO}_{2}$ and $\mathrm{H}_{2} \mathrm{~S}$ production. The API ZYM test showed that L. plantarum LRCC5314 was positive for the activities of alkaline phosphatase, leucine arylamidase, valine arylamidase, cystine arylamidase, acid phosphatase, naph-thol-AS-BIphosphohydrolase, $\beta$-galactosidase, a-glucosidase, $\beta$-glucosidase, and $\mathrm{N}$-acetyl- $\beta$-glucosaminidase.

\section{Genomic characterization}

The whole-genome sequence of L. plantarum LRCC5314 consisted of 3,249,803 bp. The chromosome contained 16 rRNAs (5S rRNA, 6; 16S rRNA, 5; $23 \mathrm{~S}$ rRNA, 5), 67 tRNAs, and 3,031 protein-coding sequences (CDSs). The G+C content of L. plantarum LRCC5314 was 44.5 mol\% (Table 1). WebMGA online tools were used to analyze L. plantarum LRCC5314 protein functions annotated in the COG database. The annotated proteins were related to the categories of translation, ribosomal structure and biogenesis ( $\mathrm{J} ; 147$ genes), transcription ( $\mathrm{K} ; 259$ genes), replication, recombination, and repair ( $\mathrm{L} ; 146$ genes), cell cycle control, cell division, and chromosome partitioning ( $\mathrm{D} ; 23$ genes), defense mechanisms ( $V ; 62$ genes), signal trans-duction mechanisms ( $T ; 72$ genes), cell wall/membrane/envelope biogenesis (M; 174 genes), intracellular trafficking, secretion, and vesicular transport (U; 22 genes), post-translational 
modification, protein turnover, and chaperones ( $0 ; 61$ genes), energy pro-duction and conversion ( $\mathrm{C} ; 106$ genes), carbohydrate transport and metabolism (G; 268 genes), amino acid transport and metabolism (E, 199 genes), nucleotide transport and metabolism ( $\mathrm{F} ; 86$ genes), coenzyme transport and metabolism ( $\mathrm{H}$; 64 genes), lipid transport and metabolism (l; 55 genes), inorganic ion transport and metabolism ( $\mathrm{P} ; 128$ genes), and secondary metabolite biosynthesis, transport, and catabolism (Q; 17 genes; Fig. S1).

RAST analysis annotated genes to assign them to the categories of amino acids and derivatives (15.8\%), cofactors, vitamins, prosthetic groups, and pigments (9.5\%), and nucleosides and nucleotides (8.0\%). Genes in the amino acids and derivatives category included those related to "glutamine, glutamate, aspartate, asparagine, and ammonia assimilation" (19 genes), "histidine metabolism" (8 genes), "arginine, urea cycle, and polyamines" (3 genes), and "lysine, threonine, methionine, and cysteine" (82 genes). Genes in the cofactors, vitamins, prosthetic groups, and pigments category included those related to "biotin" (3 genes), "riboflavin, FMN, and FAD" (20 genes), "pyridoxine" (8 genes), "NAD and MADP" (9 genes), "folate and pterines" (43 genes), "lipoic acid" (3 genes), and "co-enzyme A". Genes in the nucleosides and nucleotides category included those related to "pyrimidines" (25 genes), "purines" (47 genes), and "detoxification" (6 genes; Table S1).

\section{Comparative genomic analysis}

OrthoANI analysis between L. plantarum LRCC5314 and closely related Lactobacillus strains showed similarities in the range of $75.14-99.65 \%$. L. plantarum LRCC5314 was the most closely related to $L$. plantarum ATCC $14917^{\top}$ (99.65\%), followed by L. paraplantarum DSM $10667^{\top}$ (85.90\%). L. pentosus DSM $2031^{\top}$, L. herbarum NBRC $10733^{\top}$, and L. plajomi NBRC $10733^{\top}$ showed similarities of $79.80 \%$, $77.18 \%$, and $74.77 \%$, respectively (Fig. $2 A$ ). The dDDH value with L. plantarum ATCC $14917^{\top}$ was $97 \%$. OrthoANI and dDDH values showed that the LRCC5314 strain belonged to the species L. plantarum. Furthermore, based on BLAST comparison, the whole-genome sequences of L. plantarum LRCC5314 and L. plantarum ATCC $14917^{\top}$ showed high similarity (Fig. 2B). However, a circular comparison of the genomes of L. plantarum LRCC5314 and L. plantarum ATCC $14917^{\top}$ showed differences in the GC content and genomic similarities between the two strains. Furthermore, L. plantarum LRCC5314 contained a CRISPR in its genome. According to CRISPRFinder, L. plantarum LRCC5314 had a CRISPR without Cas genes between 1,536,174 bp and 1,536,259 bp, whereas no confirmed CRISPRs were detected in L. plantarum ATCC $14917^{\top}$. These results demonstrated that the LRCC5314 strain belonged to the same species (L. plantarum) as the ATCC $14917^{\top}$ strain but had genetic differences.

\section{Carbon source metabolic pathway}

The metabolic pathways of carbon sources related to the genes of L. plantarum LRCC5314 were combined and mapped in one synthesis pathway (Fig. 3). For carbo-hydrate metabolism, 228 genes of $L$. plantarum LRCC5314 were mapped to the KEGG pathway, and only the metabolic pathways of carbon sources with a positive result in the API $50 \mathrm{CH}$ test were combined. L. plantarum LRCC5314 could utilize various carbon sources, including glucose, sucrose, maltose, and lactose. According to the carbon source 
metabolic pathway, L. plantarum LRCC5314 could produce pyruvate from glucose via glycolysis and could not produce alcohol. These findings revealed the homofermentative activity $L$. plantarum LRCC5314, which could metabolize hexoses via the Embden-Meyerhof-Parnas pathway.

\section{Tryptophan metabolism and serotonin production}

According to gene annotation, the L. plantarum LRCC5314 genome contained a complete biosynthetic pathway for producing tryptophan from chorismate (Fig. 4 and Table S2). Tryptophan is an essential amino acid for humans because it is not synthesized in the human body, and humans need to consume it. However, only $1 \%$ of the available tryptophan is used for protein synthesis, and $99 \%$ is used as a precursor of tryptamine, melatonin, and serotonin.

\section{Tryptophan metabolism and serotonin production}

To determine the stimulatory effects of L. plantarum LRCC5314 on 5-hydroxytryptamine (5-HT) production, Caco-2 and HT-22 cells were treated with the bacterial supernatant, and the content of 5-HT was measured with the ELISA method. Caco- 2 cells were used as an in vitro model of the intestinal epithelial barrier. In the in vitro experiment with Caco-2 cells, 5-HT secretion was increased more than 1.5 times in the cell supernatant of treated Caco-2 cells compared with untreated cells (untreated versus treated Caco-2 cells: $7.2 \pm 0.15$ versus $12.8 \pm 0.7 \mathrm{pg} / \mathrm{mL}, \mathrm{p}<0.001$; Fig. 5A). The secretion of 5-HT from HT-22 cells treated with the bacterial supernatant was al-so increased with statistical significance (untreated versus treated HT-22 cells: $17.5 \pm 1.12$ versus $19.9 \pm 0.03 \mathrm{pg} / \mathrm{mL}, \mathrm{p}<0.05$; Fig. 5B). In comparison with untreated cells, Caco-2 and HT-22 cells treated with the L. plantarum LRCC 5314 supernatant showed a statistically significant increase in $5-\mathrm{HT}$ secretion. These results suggest that a component of the bacterial supernatant stimulated 5-HT secretion.

\section{Discussion}

L. plantarum is one of the probiotics commonly found in various fermented dairy products or traditional fermented foods such as kimchi or sausage [3], and its safety and beneficial effects on human health have been established. This bacterium is a versatile species that can be isolated from various environments, including fermented vegetables [16]. OrthoANI analysis showed that $L$. plantarum LRCC5314 was the most closely related to L. plantarum ATCC 14917 T with a similarity of $99.65 \%$. The $\mathrm{G}+\mathrm{C}$ content range of the $L$. plantarum species has been reported to be $44.37-44.71 \mathrm{~mol} \%$ [17], and L. plantarum LRCC5314 had a numerical value coinciding with the average [18]. These results indicated the LRCC5314 strain belonged to the L. plantarum species.

According to the API test, the LRCC5314 strain showed a positive result for $\beta$-galactosidase. $\beta$ galactosidase, also known as lactase, is an enzyme that catalyzes lactose into glucose and galactose. People who lack this enzyme can suffer from lactose intolerance and may be unable to consume dairy products [19]. However, probiotic strains with $\beta$-galactosidase can support lactose digestion in the small intestine. Bile salts from the gallbladder stimulate the lactase activity of probiotics and mitigate lactose 
intolerance symptoms [20]. L. plantarum LRCC5314 was positive for the activation of the $\beta$-galactosidase enzyme in the API test, suggesting that the strain can be used as a probiotic to alleviate lactose intolerance in the human intestine.

Hexoses are monosaccharides that contain six carbon atoms, such as mannose, glucose, and fructose. $L A B$ can be classified as homofermentative and heterofermentative based on how they metabolize hexoses. Heterofermentative LAB metabolize hexoses via the acetyl-phosphate and phosphoketolase pathway, whereas homofermentative LAB metabolize hexoses via the Embden-Meyerhof-Parnas pathway [21]. L. plantarum species, including L. plantarum LRCC5314, are homofermentative LAB and metabolize hexoses to pyruvate, lactate, carbon dioxide, and ethanol through the Embden-Meyerhof-Parnas pathway. L. plantarum LRCC5314 could utilize D-galactose, D-glucose, D-fructose, and D-mannose according to the API $50 \mathrm{CH}$ test and could metabolize them via glycolysis, which was verified by in silico analysis.

The European Food Safety Authority (EFSA) recommends that bacterial strains with antibiotic resistance genes should not be used as probiotics for humans or as an additive to animal feeds [22]. The drug resistance genes of probiotic strains have been a focus of re-search because of their risk of transfer to other microbial species in the human intestine [23]. ResFinder was used to detect genes conferring antibiotic resistance in the $L$. plantarum LRCC5314 genome, and none were detected. The gene search results showed that $L$. plantarum LRCC5314 may be safe as a potential probiotic strain without risk of antibiotic gene transfer.

In our in vitro experiment, the bacterial supernatant promoted the secretion of serotonin in Caco-2 and HT22 cells. 5-HT regulates the digestive process at several levels within the human gastrointestinal system. When foods enter the gastrointestinal tract, digestion is carried out through peristaltic waves, which are modulated by $5-\mathrm{HT}$ [24]. The HT-22 cell line is an immortalized mouse hippocampal cell line and is used to identify the release of 5-HT at the brain level. The hippocampus is part of the limbic system and plays an important role in the conversion of short-term memories to long-term memories and emotional regulation [25]. Furthermore, hippocampal cells have the 5-HT receptor, the 5-HT transporter (5-HTT), and tryptophan hydroxylase (the rate-limiting enzyme in 5-HT pro-duction), which were discovered in these cells previously [26].

Serotonin, also known as 5-HT, is synthesized via a short pathway from L-tryptophan, in which tryptophan hydroxylase and aromatic L-amino acid decarboxylase are involved [27]. This monoamine neurotransmitter has multifaceted and complex biological functions. 5-HT has been reported to play functional roles in cognition, mood modulation, learning, memory, and various physiological processes [28]. Around $90 \%$ of $5-\mathrm{HT}$ is found in the gastrointestinal tract, especially in enterochromaffin cells. $5-\mathrm{HT}$ secreted from enterochromaffin cells acts in synergy with other digestive hormones to control intestinal motility. However, $1-2 \%$ of serotonin is produced in nerve cells, such as neurons with the 5-HT receptor. 5$\mathrm{HT}$ produced at the end of neurons is released to the synapse and serves to transmit signals to other neurons [29]. This process is also known to be involved in the regulation of stress hormones such as 
cortisol, and a deficiency in 5-HT can cause depression and bipolar disorder. Therefore, several antidepressants function to prevent the absorption of 5-HT [30].

However, 5-HT cannot directly cross the blood-brain barrier (BBB). For the biosynthesis of serotonin in the brain, a precursor needs to be transported into the BBB. Tryptophan enters the brain through a common transporter protein and competes with other neutral amino acids for transport [31]. The amount of tryptophan entering the brain through the transporter protein is proportional to the concentration of tryptophan in the plasma. Several studies have reported that tryptophan-enriched diets could increase 5HT levels in the brain and decrease cortisol levels [32].

\section{Conclusion}

We analyzed the complete genome of L. plantarum LRCC5314 and shed light on its genomic features, carbon source metabolic pathway, and functional genes. Genomic analysis confirmed that $L$. plantarum LRCC5314 had the tryptophan biosynthetic gene, which could produce tryptophan, a precursor that can be used in 5-HT synthesis. Moreover, in vitro analysis demonstrated that L. plantarum LRCC5314 could stimulate 5 -HT se-creation in intestinal epithelial cells and serotonergic neurons. These results suggest that L. plantarum LRCC5314 may be used as a beneficial probiotic to alleviate mental stress and possibly to improve mental health.

\section{Abbreviations}

LAB: lactic acid bacteria; FAO/WHO: Food and Agriculture Organization/World Health Organization; PCR: polymerase chain reaction; NCBI: National Center for Biotechnology Information; COG: Clusters of orthologous groups; KEGG: Kyoto Encyclopedia of Genes and Genomes; dDDH: digital DNA-DNA hybridization; BRIG: Blast Ring Image Generator; MRS: De Man, Rogosa and Sharpe medium; ATCC: American Type Culture Collection; DMEM: Dulbecco's Modified Eagle Medium; ELISA: Enzyme-linked immunosorbent assay.

\section{Declarations}

\section{Availability of data and materials}

The data set of this study have been deposited to DDBJ/EMBL/GenBank International Nucleotide Sequence Database under the BioProject ID PRJNA716786.

\section{Consent for publication}

Not Applicable.

\section{Competing interests}

The authors declare that they do not have any competing interests. 


\section{Funding}

This study was supported by the Strategic Initiative for Microbiomes in Agriculture and Food, Ministry of Agriculture, Food and Rural Affairs, Republic of Korea (as part of the [multi-ministerial] Genome Technology to Business Translation Program; no. 918004-4).

\section{Authors' contributions}

Conceptualization, WK.; conducted the experiments, JJ. and SY.; Analyzed data, JJ., SY. and JHK.; writing -original draft preparation, JJ. and SY.; writing-review and editing, JHK. and WK.; visualization, JHK.; supervision, WK.; project administration, WK.; funding acquisition, WK. All authors have read and agreed to the published version of the manuscript.

\section{Acknowledgements}

The authors wish to thank Miss Jihye Back, for her technical assistant with the culture of bacterial strains.

\section{Supplementary Information}

The online version contains supplementary material available at http://doi.org/.

\section{References}

1. Oh NS, Joung JY, Lee JY, Kim Y. Probiotic and anti-inflammatory potential of Lactobacillus rhamnosus 4B15 and Lactobacillus gasseri 4M13 isolated from infant feces. PLoS One. 2018;13:e0192021.

2. Tanizawa $Y$, Tohno M, Kaminuma E, Nakamura Y, Arita M. Complete genome sequence and analysis of Lactobacillus hokkaidonensis $\mathrm{LOOC} 260^{\top}$, a psychrotrophic lactic acid bacterium isolated from silage. BMC Genomics. 2015;16:1-11.

3. Yang SJ, Lee JE, Lim SM, Kim YJ, Lee NK, Paik HD. Antioxidant and immune-enhancing effects of probiotic Lactobacillus plantarum 200655 isolated from kimchi. Food Sci. Biotechnol. 2019;28:491499.

4. Chervinets Y, Chervinets V, Shenderov B, Belyaeva E, Troshin A, Lebedev S, Danilenko V. Adaptation and probiotic potential of lactobacilli, isolated from the oral cavity and intestines of healthy people. Probiotics Antimicrob. Proteins. 2018;10:22-33.

5. Pan M, Hidalgo-Cantabrana C, Goh YJ, Sanozky-Dawes R, Barrangou R. Comparative analysis of Lactobacillus gasseri and Lactobacillus crispatus isolated from human urogenital and gastrointestinal tracts. Front. Microbiol. 2020;10:3146.

6. Assadi MM, Pourahmad R, Moazami N. Use of isolated kefir starter cultures in kefir production. World J. Microbiol. Biotechnol. 2000;16:541-543. 
7. Buchin S, Duboz G, Salmon JC. Lactobacillus delbrueckii subsp. lactis as a starter culture significantly affects the dynamics of volatile compound profiles of hard cooked cheeses. Eur. Food Res. Technol. 2017;243:1943-1955.

8. Song S, Lee SJ, Park DJ, Oh S, Lim KT. The anti-allergic activity of Lactobacillus plantarum L67 and its application to yogurt. J. Dairy Sci. 2016;99:9372-9382.

9. Hill C, Guarner F, Reid G, Gibson GR, Merenstein DJ, Pot B, Morelli L, Canani RB, Flint HJ, Salminen S, et al. The International Scientific Association for Probiotics and Prebiotics consensus statement on the scope and appropriate use of the term probiotic. Nat. Rev. Gastroenterol. Hepatol. 2014;11:506514.

10. Kim DH, Jeong D, Kang IB, Kim H, Song KY, Seo KH. Dual function of Lactobacillus kefiri DH5 in preventing high-fat-diet-induced obesity: direct reduction of cholesterol and upregulation of PPAR-a in adipose tissue. Mol. Nutr. Food Res. 2017;61:1700252.

11. Li M, Lee K, Hsu M, Nau G, Mylonakis E, Ramratnam B. Lactobacillus-derived extracellular vesicles enhance host immune responses against vancomycin-resistant enterococci. BMC Microbiol. 2017;17:1-8.

12. Lee E, Jung SR, Lee SY, Lee NK, Paik HD, Lim SI. Lactobacillus plantarum strain Ln4 attenuates dietinduced obesity, insulin resistance, and changes in hepatic mRNA levels associated with glucose and lipid metabolism. Nutrients. 2018;10:643.

13. Chong HX, Yusoff NAA, Hor YY, Lew LC, Jaafar MH, Choi SB, Yusoff MSB, Wahid N, Abdullah MFIL, Zakaria N, et al. Lactobacillus plantarum DR7 alleviates stress and anxiety in adults: a randomised, double-blind, placebo-controlled study. Benef. Microbes. 2019;10:55-373.

14. Ribbera A, Jï HM, Kant R, Pietilï TE, Randazzo C, Paulin L, Laine PK, Caggia C, von Ossowski I, Ruenanen J, et al. Comparative genomic and functional analysis of Lactobacillus casei and Lactobacillus rhamnosus strains marketed as probiotics. Appl. Environ. Microbiol 2013;79:19231933.

15. Stewart PS, Costerton JW. Antibiotic resistance of bacteria in biofilms. The lancet. 2001;358:135138.

16. Crowley S, Bottacini F, Mahony J, van Sinderen D. Complete genome sequence of Lactobacillus plantarum strain 16, a broad-spectrum antifungal-producing lactic acid bacterium. Genome Announc. 2013;1:e00533-13.

17. Yu J, Ahn S, Kim K, Caetano-Anolles K, Lee C, Kang J, Cho K, Yoon SH, Kang DK, Kim H. Comparative genomic analysis of Lactobacillus plantarum GB-LP1 isolated from traditional Korean fermented food. J. Microbiol. Biotechnol. 2017;27:1419-27.

18. Zhang W, Ji H, Zhang D, Liu H, Wang S, Wang J, Wang Y. Complete genome sequencing of Lactobacillus plantarum ZLP001, a potential probiotic that enhances intestinal epithelial barrier function and defense against pathogens in pigs. Front. Physiol. 2018;9:1689.

19. Saqib S, Akram A, Halim SA, Tassaduq R. Sources of $\beta$-galactosidase and its applications in food industry. 3 Biotech. 2017;7:79. 
20. De Vrese M, Stegelmann A, Richter B, Fenselau S, Laue C, Schrezenmeir J. Probiotics-compensation for lactase insufficiency. Am. J. Clin. Nutr. 2001;73:421s-429s.

21. Gänzle MG. Lactic metabolism revisited: metabolism of lactic acid bacteria in food fermentations and food spoilage. Curr. Opin. Food Sci. 2015;2:106-117.

22. European Food Safety Authority (EFSA). Introduction of a Qualified Presumption of Safety (QPS) approach for assessment of selected microorganisms referred to EFSA-Opinion of the Scientific Committee. EFSA Jol. 2007;5:587.

23. Nawaz M, Wang J, Zhou A, Ma C, Wu X, Moore JE, Cherie Miller B, Xu J. Characterization and transfer of antibiotic resistance in lactic acid bacteria from fermented food products. Curr. Microbiol. 2011;62:1081-1089.

24. Gershon MD, Tack J. The serotonin signaling system: from basic understanding to drug development for functional GI disorders. Gastroenterology. 2007;132:397-414.

25. Hensler JG. Serotonergic modulation of the limbic system Neurosci. Biobehav. Rev. 2006;30:203214.

26. Sugden K, Tichopad A, Khan N, Craig IW, D'Souza UM. Genes within the serotonergic system are differentially expressed in human brain. BMC Neurosci. 2009;10:1-11.

27. Le Floc'h N, Otten W, Merlot E. Tryptophan metabolism, from nutrition to potential therapeutic applications. Amino acids. 2011;4:1195-1205

28. Silber BY, Schmitt JAJ. Effects of tryptophan loading on human cognition, mood, and sleep. Neurosci. Biobehav. Rev. 2010;34:387-407.

29. De-Miguel FF, Leon-Pinzon C, Noguez P, Mendez B. Serotonin release from the neuronal cell body and its long-lasting effects on the nervous system. Philos. Trans. R. Soc. B. 2015;370:20140196.

30. Kato-Kataoka A, Nishida K, Takada M, Kawai M, Kikuchi-Hayakawa H, Suda K, Rokutan K. Fermented milk containing Lactobacillus casei strain Shirota preserves the diversity of the gut microbiota and relieves abdominal dysfunction in healthy medical students exposed to academic stress. Appl. Environ. Microbiol. 2016;82:3649-3658.

31. Höglund E, Øverli $\varnothing$, Winberg S. Tryptophan metabolic pathways and brain serotonergic activity: a comparative review. Front. Endocrinol. 2019;10:158.

32. Markus CR. Dietary amino acids and brain serotonin function; implications for stress-related affective changes. Neuromol. Med. 2008;10:247.

33. Lane DJ. 16S/23S rRNA sequencing. Nucleic acid techniques in bacterial systematics, 1991;115175.

34. Yoon SH, Ha SM, Kwon S, Lim J, Kim Y, Seo H, Chun J. Introducing EzBioCloud: a taxonomically united database of $16 \mathrm{~S}$ rRNA gene sequences and whole-genome assemblies. Int. J. Syst. Evol. Microbiol. 2017;67:1613.

35. Larkin MA, Blackshields G, Brown NP, Chenna R, McGettigan PA, McWilliam H, Wallace IM, Wilm A, Lopez R, Gibson TJ, et al. Clustal W and Clustal X version 2.0. Bioinformatics. 2007;23:2947-2948. 
36. Felsenstein J. Evolutionary trees from DNA sequences: a maximum likelihood approach. J. Mol. Evol, 1981;17:368-376.

37. Kumar S, Stecher G, Tamura K. MEGA7: molecular evolutionary genetics analysis version 7.0 for bigger datasets. Mol. Biol. Evol. 2016;33:1870-1874.

38. Saitou N, Imanishi T. Relative efficiencies of the Fitch-Margoliash, maximum-parsimony, maximumlikelihood, minimum-evolution, and neighbor-joining methods of phylogenetic tree construction in obtaining the correct tree. Mol. Biol. Evol. 1989;6:514-525.

39. Aziz RK, Bartels D, Best AA, DeJongh M, Disz T, Edwards RA, Formsma K, Gerdes S, Glass EM, Kubal $M$, et al. The RAST Server: rapid annotations using subsystems technology. BMC Genomics. 2008;9:1-15.

40. Tatusova T, DiCuccio M, Badretdin A, Chetvernin V, Nawrocki EP, Zaslavsky L, Lomsadze A, Pruitt KD, Borodovsky M, Ostell J. NCBI prokaryotic genome annotation pipeline. Nucleic Acids Res. 2016;44:6614-6624.

41. Wu S, Zhu Z, Fu L, Niu B, Li W. WebMGA: a customizable web server for fast metagenomic sequence analysis. BMC Genomics. 2011;12:444.

42. Bortolaia V, Kaas RS, Ruppe E, Roberts MC, Schwarz S, Cattoir V, Fagelhauer L. ResFinder 4.0 for predictions of phenotypes from genotypes. J. Antimicrob. Chemother. 2020;75:3491-3500.

43. Kanehisa M, Goto S, Sato Y, Kawashima M, Furumichi M, Tanabe M. Data, information, knowledge and principle: back to metabolism in KEGG. Nucleic Acids Res. 2014;42:D199-D205.

44. Johnson M, Zaretskaya I, Raytselis Y, Merezhuk Y, McGinnis S, Madden TL. NCBI BLAST: a better web interface. Nucleic Acids Res. 2008;36:W5-W9.

45. Meier-Kolthoff JP, Auch AF, Klenk HP, Göker M. Genome sequence-based species delimitation with confidence intervals and improved distance functions. BMC Bioinform. 2013;14:60.

46. Lee I, Kim YO, Park SC, Chun J. OrthoANI: an improved algorithm and software for calculating average nucleotide identity. Int. J. Syst. Evol. Microbiol. 2016;66:1100-1103.

47. Alikhan NF, Petty NK, Zakour NLB, Beatson SA. BLAST Ring Image Generator (BRIG): simple prokaryote genome comparisons. BMC Genome. 2011;12:402.

48. Grissa I, Vergnaud G, Pourcel C. CRISPRFinder: a web tool to identify clustered regularly interspaced short palindromic repeats. Nucleic Acids Res. 2007;35:W52-W57.

\section{Tables}

Table 1. The genomic characterization of L. plantarum LRCC5314 and L. plantarum ATCC $14917^{\top}$ (ACGZ02000000) was performed. CRISPR loci were analyzed using CRISPRFinder. 


\begin{tabular}{|lll|}
\hline & L. plantarum LRCC5314 & L. plantarum ATCC $14917^{\top}$ \\
\hline Genome size (bp) & $3,249,803$ & $3,212,261$ \\
\hline GC content (\%) & 44.5 & 44.6 \\
\hline Annotated genes & 3,306 & 3,011 \\
\hline tRNAs & 67 & 67 \\
\hline rRNAs & 16 & 16 \\
\hline CRISPR loci & 1 & 0 \\
\hline
\end{tabular}

\section{Figures}

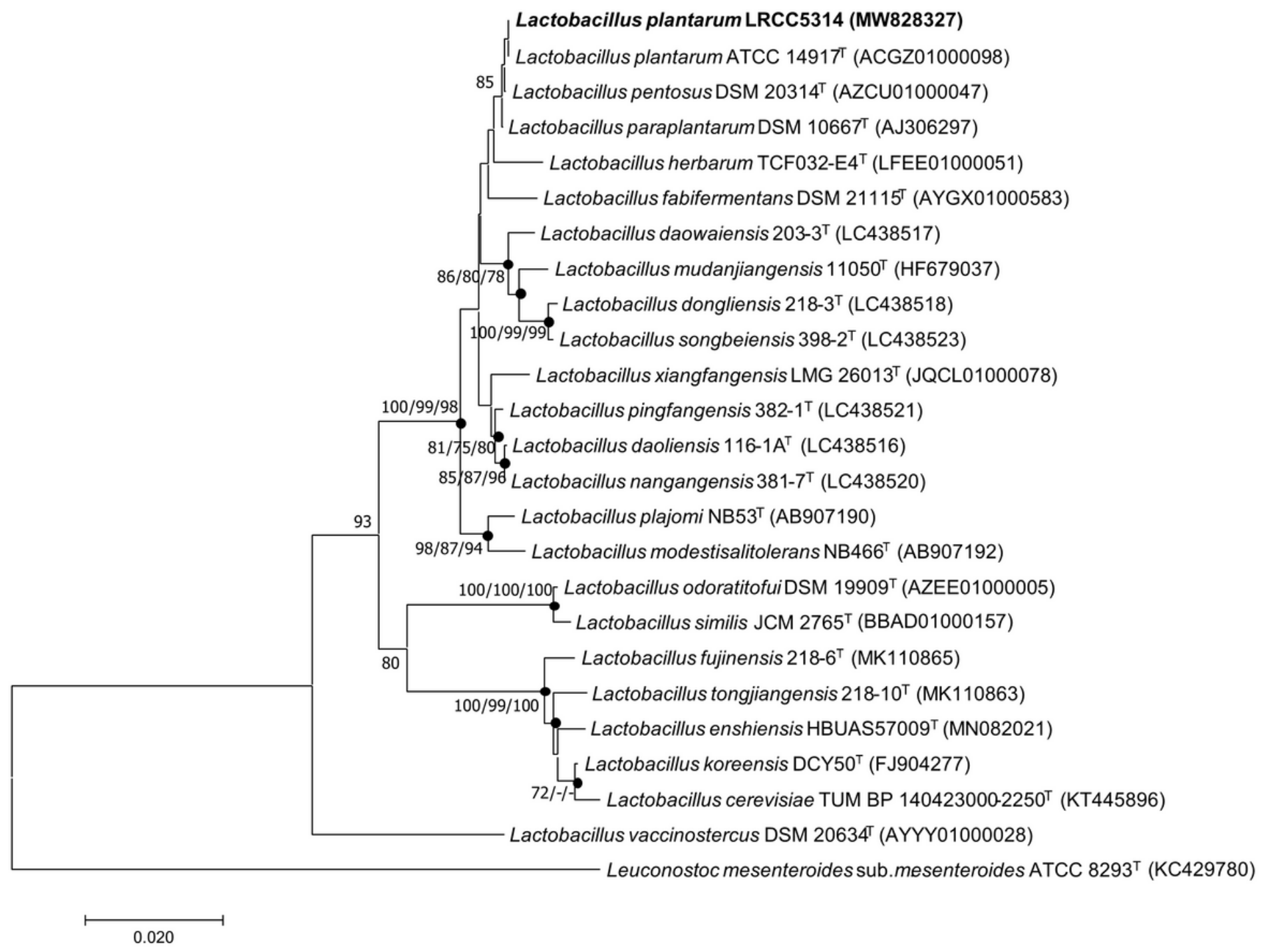

Figure 1 
Phylogenetic tree constructed with the neighbor-joining method for L. plantarum LRCC5314 and related strains of the genus Lactobacillus. The filled circle indicates that the corresponding nodes were also recovered in the maximum-likelihood and maximum-parsimony trees. The numbers at the nodes indicate the percentage of bootstrapping based on 1,000 resampling; only values $>70 \%$ are given. Bar, 0.02 substitutions per nucleotide position. Leuconostoc mesenteroides subsp. mesenteroides ATCC 8293T (KC429780) was used as an outgroup organism.

A
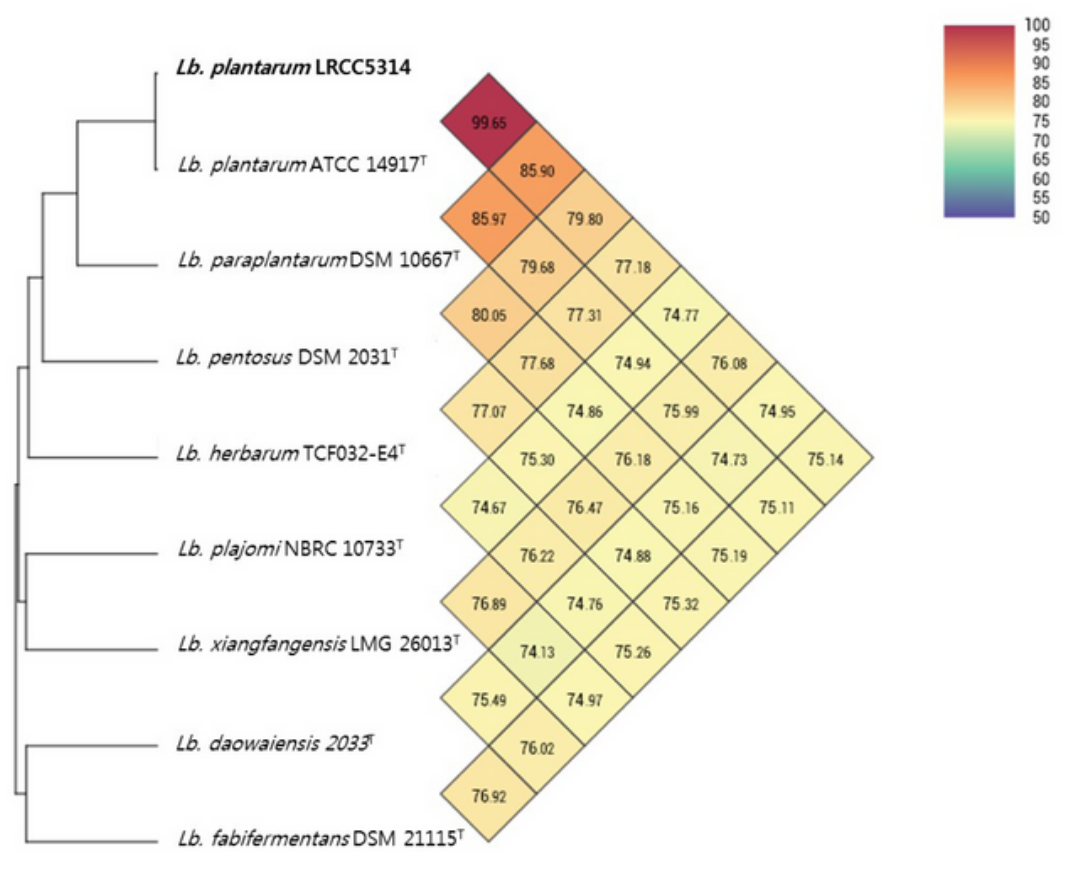

B

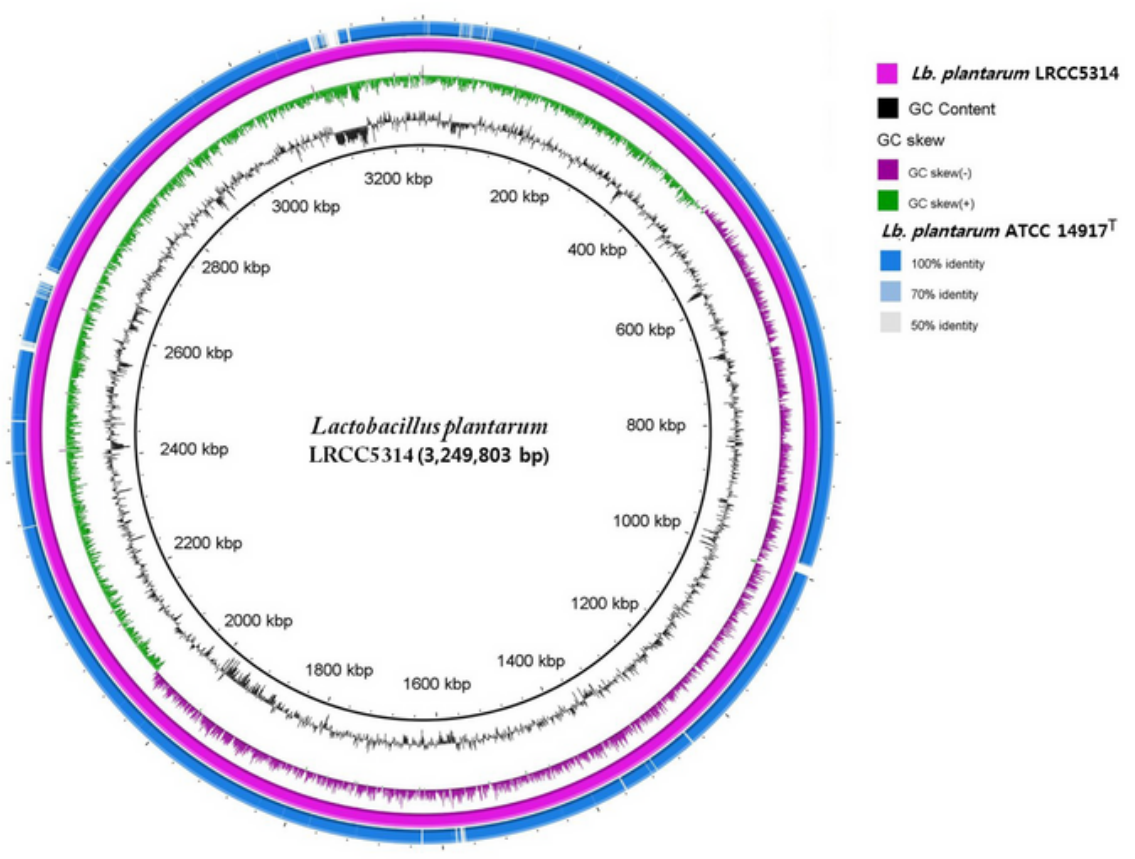

Figure 2 
Comparative genomic analysis of L. plantarum LRCC5314 and Lactobacillus strains. (A) OrthoANI values between L. plantarum LRCC5314 and closely related Lactobacillus strains (L. plantarum ATCC 14917T, L. paraplantarum DSM 10667T, L. pentosus DSM 2031T, L. herbarum TCF032-E4T, L. plajomi NBRC 10733T, L. xiangfangensis LMG 26013T, L. daowaiensis 2033T, and L. fabifermantans DSM 21115T). (B) Circular comparison of the genomes of L. plantarum LRCC5314 and L. plantarum ATCC 14917T. From outside to inside, the first circle shows the genome of L. plantarum ATCC 14917T, the second circle shows the genome of L. plantarum LRCC5314, the third circle shows the GC skew, and the fourth circle shows the GC content.

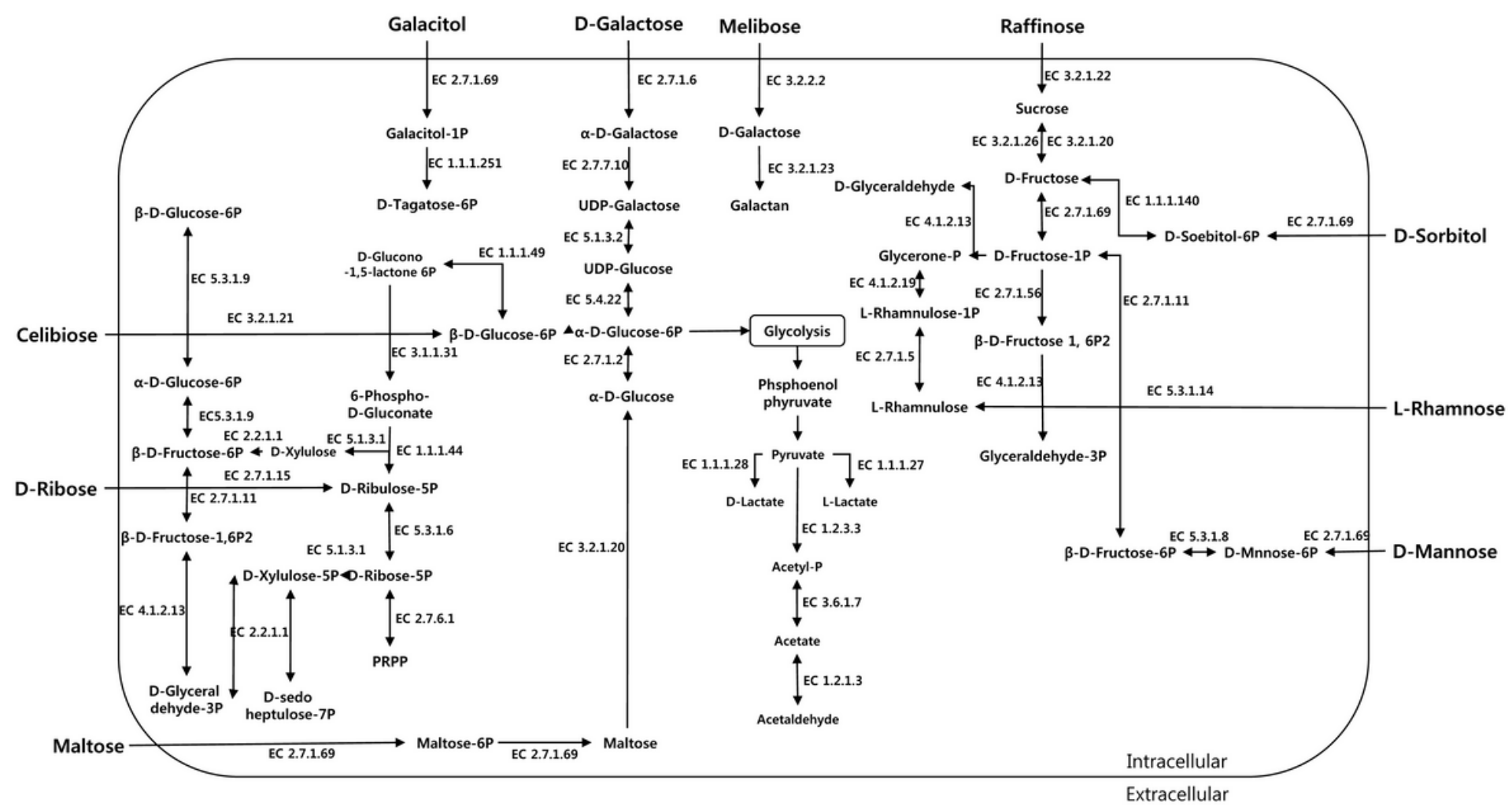

Figure 3

Carbon source metabolic pathway of L. plantarum LRCC5314. Various carbon compounds are involved in the overall metabolic pathway of L. plantarum LRCC5314. 


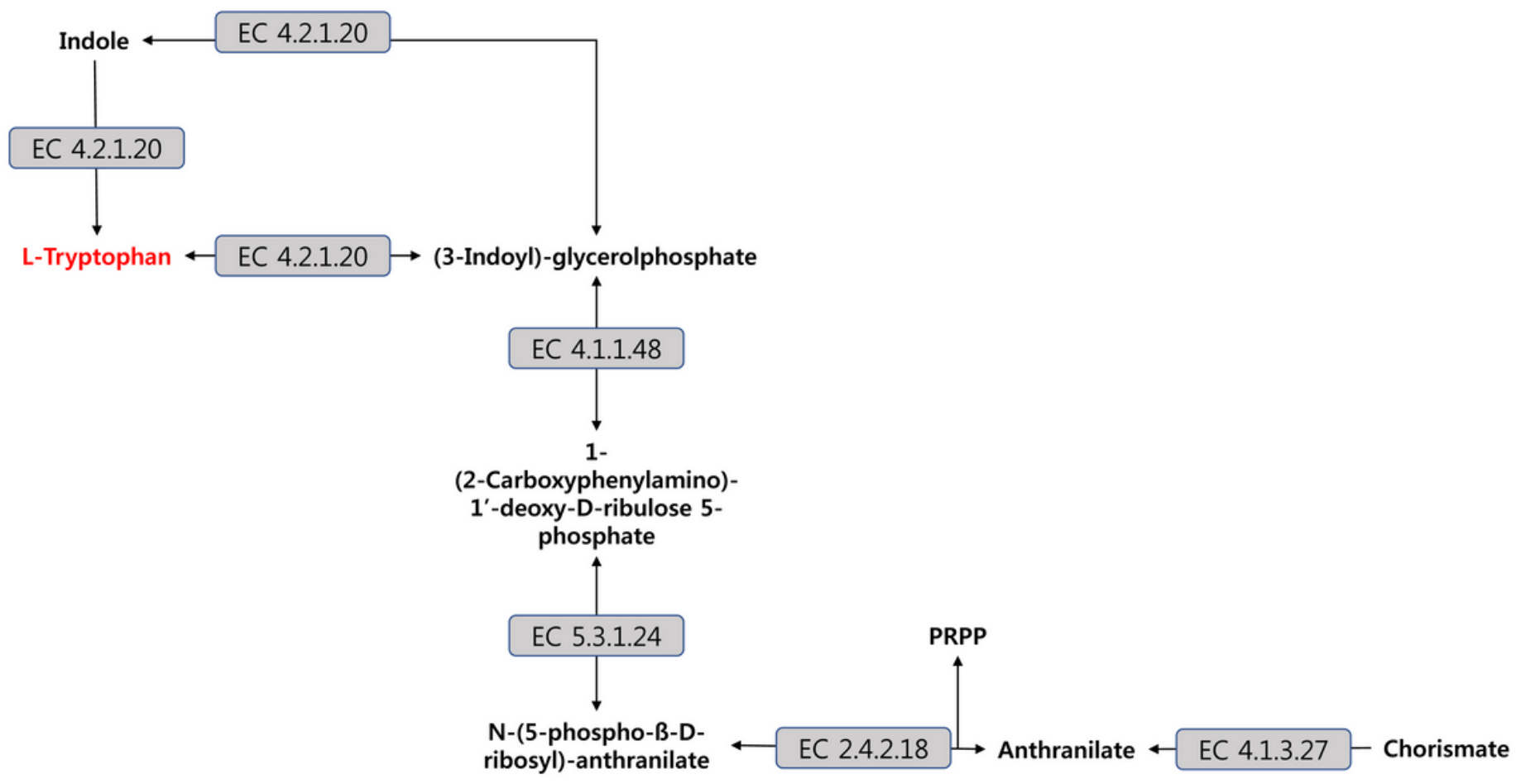

\section{Figure 4}

Tryptophan biosynthesis in L. plantarum LRCC5314. The tryptophan biosynthetic pathway and related enzymes are shown. Grey boxes indicate enzymes in the tryptophan bio-synthetic pathway present in L. plantarum LRCC5314.
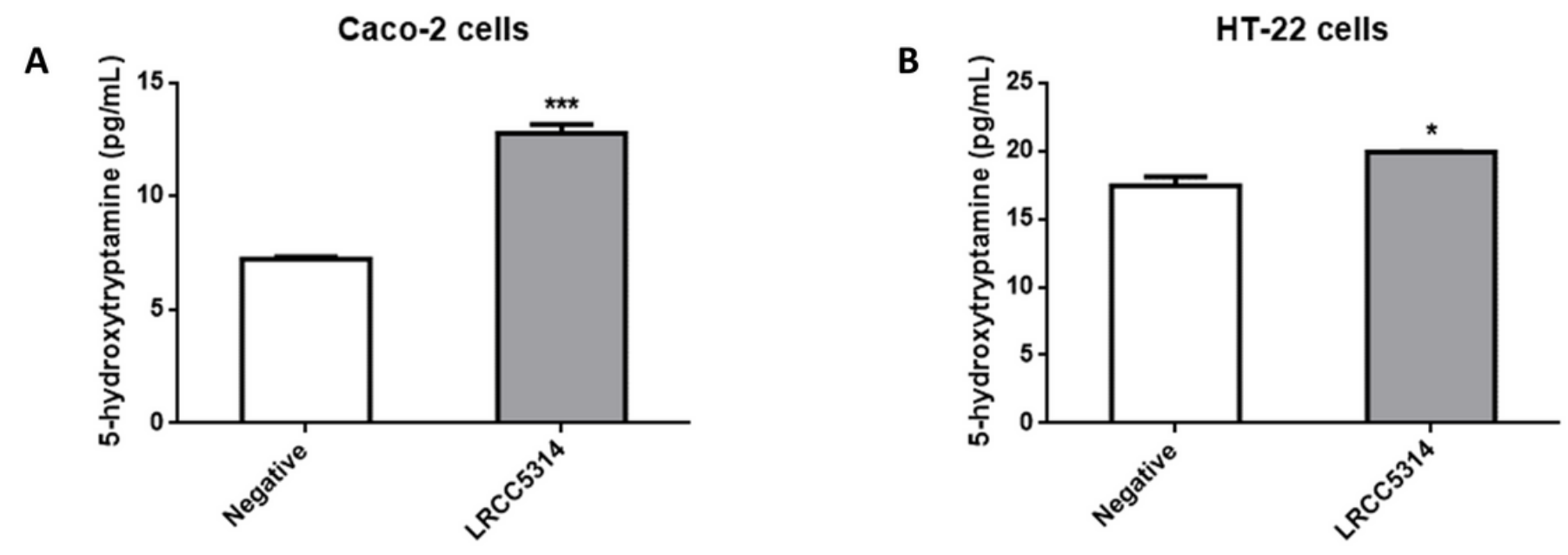

Figure 5

Concentration of 5-hydroxytryptamine secreted by the Caco-2 and HT-22 cell lines. The concentration of 5-hydroxytryptamine (5-HT) from (A) Caco-2 cells and (B) HT-22 cells was measured after treatment with 
the culture supernatant of L. plantarum LRCC5314. ${ }^{\star} p<0.05 ; * \star * p<0.001$.

\section{Supplementary Files}

This is a list of supplementary files associated with this preprint. Click to download.

- Supplementarymaterial.docx 